13.4

\title{
Генерация импульсов гигантской амплитуды в клистронном автогенераторе хаоса
}

\author{
(C) С.В. Гришин, Б.С. Дмитриев, В.Н. Скороходов \\ Саратовский национальный исследовательский государственный университет им. Н.Г. Чернышевского, Саратов, Россия \\ E-mail: sergrsh@yandex.ru
}

Поступило в Редакцию 14 июня 2019г.

В окончательной редакции 14 июня 2019г.

Принято к публикации 20 июня 2019г.

\begin{abstract}
Представлены экспериментальные результаты, демонстрирующие формирование микроволновых импульсов „гигантской“ амплитуды в клистронном генераторе, работающем в автономном режиме хаотических колебаний. Автогенератор собран по схеме шумотрона, которая содержит два последовательно соединенных в кольцо пятирезонаторных пролетных клистрона, один из которых работает в режиме линейного усиления сигнала (линейный клистрон), а другой — в режиме нелинейного усиления сигнала (нелинейный клистрон). Установлено, что при определенном значении тока пучка нелинейного клистрона в автоколебательной системе реализуется режим перемежаемости типа хаос-хаос в виде хаотических последовательностей микроволновых импульсов „гигантской“ амплитуды, формирующихся на хаотическом амплитудном фоне. Данный тип перемежаемости обусловлен амплитудной бистабильностью нелинейного пролетного клистрона.
\end{abstract}

Ключевые слова: шумотрон, пролетный клистрон, хаос, бистабильность, перемежаемость.

DOI: 10.21883/PJTF.2019.19.48315.17928

Впервые генерация хаотического СВЧ-сигнала была обнаружена в 60-х годах прошлого века в кольцевой автоколебательной системе, состоящей из двух последовательно соединенных ЛБВ-усилителей (ЛБВ лампа бегущей волны) [1,2]. В таком автогенераторе, получившем название „шумотрон“, одна из ЛБВ работала в режиме почти линейного усиления (линейная ЛБВ), а другая - в сильно нелинейном режиме, на падающем участке амплитудной характеристики (нелинейная ЛБВ). Наличие падающего участка приводило к развитию амплитудного механизма модуляции сигнала и генерации хаотических колебаний. В начале 2000-х годов появились экспериментальные и теоретические работы, в которых исследовалась хаотическая динамика кольцевой автоколебательной системы на основе другого вакуумного СВЧ-усилителя - пятирезонаторного пролетного клистрона [3,4]. В отличие от шумотрона на основе ЛБВ-усилителей в клистронном автогенераторе амплитудный механизм модуляции сигнала развивался на одной кольцевой моде, что приводило к генерации узкополосного хаотического СВЧ-сигнала. В эти же годы были проведены исследования хаотической динамики каскадного клистронного автогенератора, в котором, как и в схеме шумотрона, один из усилителей работал в режиме линейного усиления сигнала (линейный клистрон), а другой - в сильно нелинейном режиме, на падающем участке амплитудной характеристики (нелинейный клистрон) [5]. Полученные в работе [5] экспериментальные результаты касались в основном определения значений пускового тока, при которых наблюдалась сложная динамика. Здесь также было проведено сравнение полученных значений пускового тока с аналогичными значениями, полученными в хаотическом автогенераторе с одним пролетным клистроном.
Генерация же хаотических микроволновых импульсов в схемах шумотрона с ЛБВ-усилителем или пролетным клистроном, работающих в сильно нелинейном режиме, была реализована только при использовании внешнего импульсного воздействия [6,7].

В последние годы появился ряд теоретических работ, в которых была продемонстрирована генерация мощных импульсов или импульсов гигантской амплитуды в кольцевых автоколебательных системах с пассивной синхронизацией мод. Данный режим реализовался за счет использования двух ЛБВ, одна из которых работала в режиме усиления СВЧ-сигнала, а другая - в режиме его подавления $[8,9]$. В таких системах пиковая мощность генерируемых импульсов значительно превышала мощность сигнала в стационарном режиме. Кроме того, в [10] в численном эксперименте была обнаружена генерация волн-убийц в гиротроне, работающем в режиме развитого хаоса. В настоящей работе впервые демонстрируется автономная генерация микроволновых импульсов в схеме шумотрона с двумя пролетными клистронами, работающей в режиме динамического хаоса.

На рис. 1, $a$ приведена схема клистронного автогенератора, в которой используются два пятирезонаторных пролетных клистрона средней мощности типа КУ-134Е, охваченных внешней цепью положительной обратной связи. Один из клистронов-усилителей (первый клистрон) работает в режиме линейного усиления, a другой клистрон-усилитель (второй клистрон) - в сильно нелинейном режиме. Работа первого пролетного клистрона в режиме линейного усиления обеспечивается за счет использования на его входе переменного аттенюатора с ослаблением $21.5 \mathrm{~dB}$. Бо́льшая часть мощности сигнала с выхода второго клистрона-усилителя возвращается обратно в кольцо, а меньшая часть мощности 


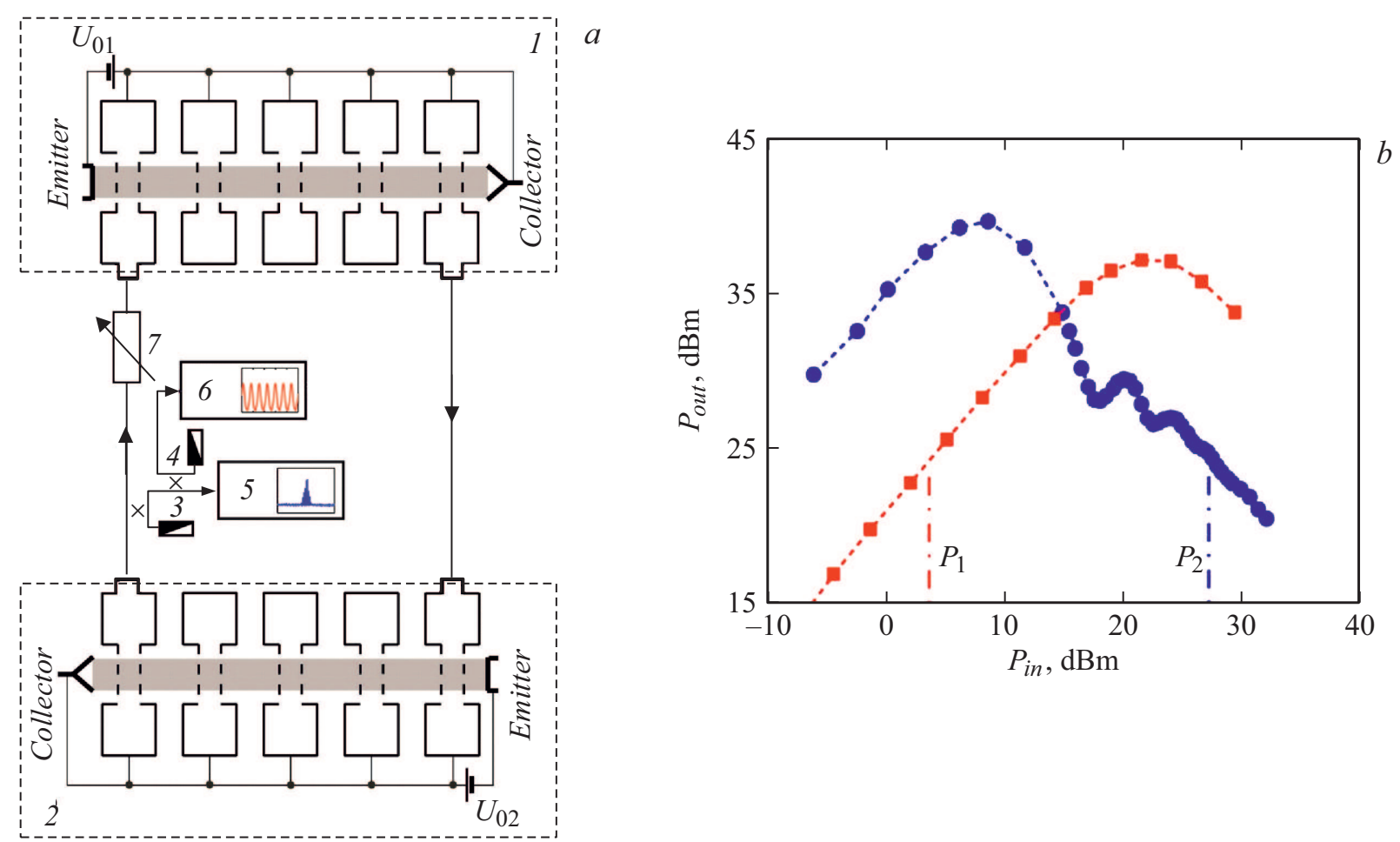

Рис. 1. $a-$ схематическое изображение клистронного автогенератора хаоса: 1,2 - первый и второй пролетные клистроны, 3,4 - направленные ответвители, 5 - анализатор спектра, 6 - осциллограф реального времени, 7 - переменный аттенюатор. $b$ - зависимости мощности сигнала на выходе от мощности сигнала на входе первого (квадраты) и второго (кружки) пролетных клистронов, измеренные на частоте $f_{0}=2797 \mathrm{MHz}$ при значениях ускоряющего напряжения $U_{01}=2200 \mathrm{~V}$ и тока пучка $I_{1}=45 \mathrm{~mA}$ первого клистрона, а также при значениях ускоряющего напряжении $U_{02}=2110 \mathrm{~V}$ и тока пучка $I_{2}=42 \mathrm{~mA}$ второго клистрона.

сигнала через микрополосковые противонаправленные ответвители поступает на входы анализатора спектра E4408В и осциллографа реального времени Infiniium DSO81004B для анализа и последующей обработки. Осциллограф реального времени характеризуется полосой пропускания $10 \mathrm{GHz}$. Для анализа временни́х рядов частота дискретизации сигнала выбиралась равной $10 \mathrm{G} / \mathrm{s}$. В этом случае глубина памяти составляла 2 млн точек. Уровень мощности сигнала на входе линейного клистрона-усилителя регулируется с помощью переменного аттенюатора.

На рис. 1, $b$ приведены зависимости мощности сигнала на выходе от мощности сигнала на входе первого и второго пролетных клистронов, измеренные на частоте, находящейся вблизи частоты генерации монохроматического СВЧ-сигнала в кольце. На этом же рисунке штрихпунктирными линиями показаны уровни мощности СВЧ-сигнала на входе первого $\left(P_{1}=+3.5 \mathrm{dBm}\right)$ и второго $\left(P_{2}=+27.2 \mathrm{dBm}\right)$ клистронов, при достижении которых в кольцевом автогенераторе наблюдается формирование микроволновых импульсов гигантской амплитуды. Видно, что в этом случае первый пролетный клистрон работает в режиме линейного усиления, а второй - в сильно нелинейном режиме. Данный режим начинается со значения входной мощности $P_{\text {in }} \cong+8 \mathrm{dBm}$. При превышении этого уровня на характеристике $P_{\text {out }}\left(P_{\text {in }}\right)$ второго клистрона возникает падающий участок из-за перегруппировки электронов в пучке. Уменьшение мощности СВЧ-сигнала на выходе данного клистрона наблюдается вплоть до уровня входной мощности $P_{\text {in }}=+18 \mathrm{dBm}$, при превышении которого на характеристике $P_{\text {out }}\left(P_{i n}\right)$ вновь появляется участок подъема. Второй падающий участок начинается при $P_{\text {in }}=+20.5 \mathrm{dBm}$ и сменяется участком подъема при $P_{i n}>+22.5 \mathrm{dBm}$. Третий падающий участок наблюдается при $P_{i n}>+24 \mathrm{dBm}$. Из представленных на рис. $1, b$ экспериментальных результатов следует, что первый пролетный клистрон работает в режиме линейного усиления, а второй пролетный клистрон - в режиме нелинейного усиления, причем сложная динамика взаимодействия электронного пучка с высокочастотным полем большой амплитуды приводит к формированию на характеристике $P_{\text {out }}\left(P_{\text {in }}\right)$ второго клистрона двух $N$-образных участков. Таким образом, второй пролетный клистрон является не только нелинейным, но и бистабильным элементом.

При проведении экспериментальных исследований режимов генерации клистронного автогенератора был зафиксирован переход к хаосу через амплитудный механизм модуляции сигнала. Эксперимент проводился при изменении тока пучка нелинейного клистронаусилителя. Так, при токе пучка второго клистрона $I_{2}=36.5 \mathrm{~mA}$ в кольце генерировался монохроматический сигнал на частоте доминантной кольцевой моды с 

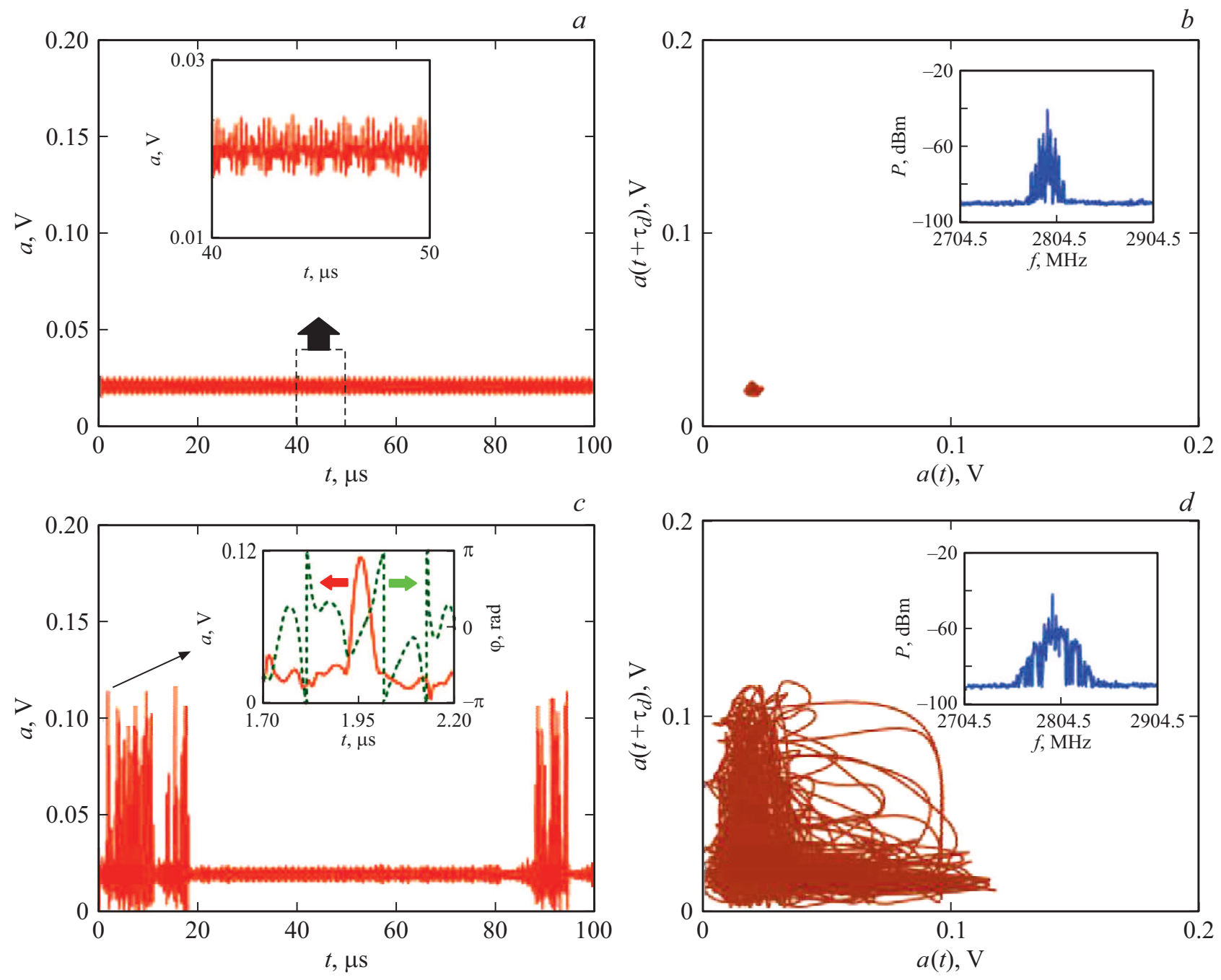

Рис. 2. Временны́е реализации $(a, c)$ и проекции фазового портрета $(b, d)$ на плоскость параметров $\left(a(t), a\left(t+\tau_{d}\right)\right)$ огибающей сигнала, генерируемого в одномодовом режиме при двух значениях тока пучка второго клистрона $I_{2}=41(a, b)$ и $42 \mathrm{~mA}(c, d)$. Данные получены при значениях тока пучка $I_{1}=45 \mathrm{~mA}$ и ускоряющего напряжения $U_{01}=2200 \mathrm{~V}$ первого клистрона, а также при значении ускоряющего напряжения $U_{02}=2110 \mathrm{~V}$ второго клистрона. На вставках показаны увеличенные фрагменты временны́х реализаций $(a, c)$ и спектры мощности генерируемого СВЧ-сигнала $(b, d)$. На частях $b, d \tau_{d}-$ временной сдвиг, равный $100 \mathrm{~ns}$.

мощностью $P=+16 \mathrm{dBm}$, измеренной на входе второго клистрона. Увеличение тока пучка до $I_{2}=38 \mathrm{~mA}$ приводило к развитию амплитудного механизма модуляции сигнала в кольце. В спектре сигнала появлялись сателлиты, отстроенные от несущей на величину $\Omega_{0} \approx 4 \mathrm{MHz}$. При токе пучка $I_{2}=40 \mathrm{~mA}$ помимо сателлитов с частотой $\Omega_{0}$ (основные сателлиты) в спектре сигнала возникали дополнительные сателлиты, отстроенные от основных сателлитов на величину $\Omega_{1} \approx 1 \mathrm{MHz}$. При этом спектральные линии дополнительных сателлитов были „размытыми“. Во временной области наблюдалось увеличение глубины амплитудной модуляции, которая становилась хаотической.

На рис. 2, $a, b$ приведены экспериментальные результаты (временной ряд, фазовый портрет огибающей и спектр мощности СВЧ-сигнала), полученные при токе пучка $I_{2}=41 \mathrm{~mA}$. В этом случае между основными и дополнительными сателлитами возникают сателлиты с частотами $\Omega_{1} / 2$. Одновременно с этим происходит увеличение шумового пьедестала в спектре генерируемого СВЧ-сигнала (см. вставку на рис. 2,b). Во временной области (рис. $2, a)$ глубина модуляции огибающей ${ }^{1}$ возрастает, при этом сама модуляция остается хаотической (см. вставку на рис. 2,a). В фазовом пространстве (рис. 2,b) формируется аттрактор, напоминающий по виду неподвижную точку, которая, однако, является сильно увеличенной в размерах из-за нерегулярного движения фазовых траекторий.

На рис. 2, $c, d$ представлены экспериментальные результаты, соответствующие режиму перемежаемости типа хаос-хаос. Данный режим наблюдается при токе

\footnotetext{
${ }^{1}$ Необходимо отметить, что амплитуда и фаза огибающей рассчитывались по временны́м рядам с СВЧ-заполнением, к которым сначала применялась цифровая обработка для фильтрации шумов квантования, а затем - преобразование Гильберта.
} 
пучка $I_{2}=42 \mathrm{~mA}$ и сопровождается формированием хаотических последовательностей импульсов гигантской амплитуды на хаотическом амплитудном фоне. Как следует из результатов, представленных на рис. 2,c, на хаотическом амплитудном фоне появляются цуги колебаний достаточно большой амплитуды, временны́е интервалы существования которых перемежаются с временны́ми интервалами, на которых указанные структуры отсутствуют. Цуги колебаний содержат импульсы, пиковые значения которых существенно превышают значение амплитудного пьедестала. Амплитудный и фазовый профиль одного из таких импульсов показан на вставке к рис. 2, c. Измерения пиковой мощности показали, что она превышает среднюю мощность генерируемого хаотического сигнала на величину $\sim 13 \mathrm{~dB}$, т.е. максимальное значение пиковой мощности $P_{p} \cong 40 \mathrm{dBm}$ соответствует мощности насыщения второго клистрона. В связи с этим данные импульсы можно называть импульсами гигантской амплитуды. Длительность отдельно взятого импульса, измеренная по половинному уровню амплитуды от ее максимального значения, составляет величину $T_{d} \cong 55 \mathrm{~ns}$, что не превышает времени прохождения (времени задержки) сигнала через клистрон-усилитель. Фаза внутри импульса имеет линейное нарастание во времени, что характерно для сигналов с линейной частотной модуляцией. Генерируемые импульсные последовательности характеризуются усредненным на длине реализации периодом следования $T_{r} \cong 25 \mu \mathrm{s}$. Как следует из результатов, представленных на вставке к рис. $2, d$, спектр мощности сигнала является нестабильным во времени, так как в нем присутствуют нестабильные во времени спектральные компоненты, частотный интервал между которыми определяет период следования импульсов. В фазовом пространстве (рис. 2,d) формируется новый хаотический аттрактор, который представляет собой нерегулярные переходы фазовой траектории из области малых значений амплитуды в область больших значений амплитуды. Таким образом, в исследуемом нами клистронном автогенераторе реализуется перемежаемость типа хаос-хаос, которая связана с наличием у нелинейного элемента бистабильности [11]. Необходимо отметить, что формирование хаотических импульсов на хаотическом амплитудном фоне наблюдалось ранее в бистабильной полупроводниковой системе с вольтамперной характеристикой $S$-типа, в которой исследовалось явление детерминированного стохастического резонанса [12].

Дальнейшее увеличение тока пучка второго клистрона приводило к генерации хаотического сигнала со сплошным спектром, форма которого являлась колоколообразной. Во временной области квазипериод следования хаотических микроволновых импульсов уменьшался, а в фазовом пространстве наблюдалось увеличение числа траекторий вдоль диагонали фазовой плоскости.

В заключение отметим, что полученные в работе результаты могут быть реализованы и в более высокочастотном диапазоне при использовании в схеме шумотрона, например, гироклистронов [13,14]. Последнее является актуальным для создания источников импульсных сигналов в коротковолновой части микроволнового диапазона частот, что может найти применение для систем связи и локации.

\section{Благодарности}

Авторы выражают благодарность Д.И. Трубецкову и О.И. Москаленко за обсуждение полученных результатов и ценные замечания.

\section{Финансирование работы}

Работа выполнена за счет гранта Российского фонда фундаментальных исследований (проект № 18-0200666).

\section{Конфлликт интересов}

Авторы заявляют, что у них нет конфликта интересов.

\section{Список литературы}

[1] Анисимова Ю.В., Воронцов Г.М., Залогин Н.Н., Кислов В.Я., Мясин Е.А. // Радиотехника. 2000. № 2. С. 19-25.

[2] Кислов В.Я., Мясин Е.А., Богданов Е.В. А.с. № 1125735 (СССР). 23.11.1984. Бюл. № 43.

[3] Дмитриев Б.С., Жарков Ю.Д., Рыскин Н.М., Шигаев А.М. // Радиотехника и электроника. 2001. Т. 46. № 5. C. 604-610.

[4] Дмитриев Б.С., Жарков Ю.Д., Клокотов Д.В., Рыскин Н.М. // ЖТФ. 2003. Т. 73. В. 7. С. 105-110.

[5] Дмитриев Б.С., Жарков Ю.Д., Скороходов В.Н., Семеновых П.Ю., Бирюков А.А. // ЖТФ. 2005. Т. 75. В. 12. С. 94 97.

[6] Дмитриев Б.С., Жарков Ю.Д., Скороходов В.Н., Геншафт А.M. // Изв. вузов. Прикладная нелинейная динамика. 2007. Т. 15. № 3. С. 52-57.

[7] Гришин С.В., Дмитриев Б.С., Жарков Ю.Д., Манышев Р.А., Скороходов В.Н. // Изв. вузов. Прикладная нелинейная динамика. 2012. Т. 20. № 5. С. 137-155.

[8] Ginzburg N.S., Denisov G.G., Vilkov M.N., Zotova I.V., Sergeev A.S. // Phys. Plasmas. 2016. V. 23. N 5. P. 050702.

[9] Ginzburg N.S., Denisov G.G., Vilkov M.N., Sergeev A.S., Zotova I.V., Samsonov S.V., Mishakin S.V. // Phys. Plasmas. 2017. V. 24. N 2. P. 023103.

[10] Ginzburg N.S., Rozental R.M., Sergeev A.S., Fedotov A.E., Zotova I.V., Tarakanov V.P. // Phys. Rev. Lett. 2017. V. 119. N 3. P. 034801.

[11] Анищенко В.С., Астахов В.В., Вадивасова Т.Е., Нейман А.Б., Стрелкова Г.И., Шиманский-Гайер Л. Нелинейные эффекты в хаотических и стохастических системах. М.-Ижевск: Ин-т компьютерных исследований, 2003. $544 \mathrm{c}$.

[12] Камилов М.К., Алиев К.М., Ибрагимов Х.О., Абакарова Н.С. // Письма в ЖТФ. 2004. Т. 30. В. 4. С. 25-32.

[13] Rozental R.M., Zotova I.V., Ginzburg N.S., Sergeev A.S., Tarakanov V.P. // IEEE Trans. Plasma Sci. 2018. V. 46. N 7. P. 2470-2474.

[14] Розенталь Р.М., Исаева О.Б., Гинзбург Н.С., Зотова И.В., Рожнев А.Г., Тараканов В.П., Сергеев А.С. // Изв. вузов. Прикладная нелинейная динамика. 2018. Т. 26. № 3. С. 78 98. 